\title{
Indication for percutaneous aortic valve implantation
}

Ibrahim Akin*, Stephan Kische*, Tim C. Rehders, Christoph A. Nienaber, Mathias Rauchhaus, Hüseyin Ince, Henrik Schneider, Andreas Liebold

Department of Medicine I, Divisions of Cardiology, Pulmonology and Intensive Care Unit at the University Hospital Rostock, Rostock School of Medicine, Rostock, Germany

Submitted: 7 November 2009

Accepted: 24 January 2010

Arch Med Sci 2010; 6, 3: 296-302

DOI: 10.5114 /aoms.2010.14247

Copyright @ 2010 Termedia \& Banach

*Both authors contributed equally to this manuscript.

\section{Abstract}

The incidence of valvular aortic stenosis has increased over the past decades due to improved life expectancy. Surgical aortic valve replacement is currently the only treatment option for severe symptomatic aortic stenosis that has been shown to improve survival. However, up to one third of patients who require lifesaving surgical aortic valve replacement are denied surgery due to high comorbidities resulting in a higher operative mortality rate. In the past such patients could only be treated with medical therapy or percutaneous aortic valvuloplasty, neither of which has been shown to improve mortality. With advances in interventional cardiology, transcatheter methods have been developed for aortic valve replacement with the goal of offering a therapeutic solution for patients who are unfit for surgical therapy. Currently there are two catheter-based treatment systems in clinical application (the Edwards SAPIEN aortic valve and the CoreValve ReValving System), utilizing either a balloonexpandable or a self-expanding stent platform, respectively.

Key words: aortic stenosis, CoreValve, Edwards SAPIEN, EuroSCORE.

\section{Introduction}

Rising life expectancy results in an increase in degenerative and neoplastic diseases. Population-based observational studies have revealed that $1 \%$ to $2 \%$ of patients older than 65 years have moderate to severe aortic stenosis [1]. Surgical aortic valve replacement dates back to 1960 and is currently the only treatment option for severe aortic stenosis that has been shown to improve survival, regardless of age [2]. In the ideal candidate, surgical aortic valve replacement (AVR) has an estimated operative mortality of $4 \%$ [2]. Unfortunately, up to one third of patients with severe aortic stenosis (AS) are ineligible for corrective valve surgery, either because of advanced age or the presence of multiple comorbidities [3]. Current treatment options for those patients not offered surgery include medical treatment and percutaneous balloon aortic valvuloplasty, though neither has been shown to reduce mortality. Medically treated patients with symptomatic AS have a 1- and 5 -year survival of $60 \%$ and $32 \%$, respectively [4]. With the introduction of percutaneous aortic valve implantation in 2002 there seems to be an alternative for these patients.
Corresponding author: Prof. Dr. Hüseyin Ince Department of Medicine I Divisions of Cardiology, Pulmonology and Intensive Care Unit University Hospital Rostock Rostock School of Medicine Ernst-Heydemann-Str. 6 18057 Rostock, Germany Phone: +49 (0)381 4947797 Fax: +49 (0)381 4947702 E-mail: hueseyin.ince@med. uni-rostock.de 


\section{Selection of patients}

Due to the existence of the tried and tested surgical valve replacement with good long-term results the selection of patients for percutaneous aortic valve implantation (PAVI), which should be done with multidisciplinary consultation between cardiologists, surgeons, imaging specialists and anaesthesiologists, involves several critical steps [5]. Candidates considered for PAVI must have severe symptomatic aortic stenosis in addition to a formal contraindication to surgery or other characteristics that would limit their surgical candidacy because of excessive mortality or morbidity risk (Figure 1). The procedure should be offered to patients who have a potential for functional improvement after valve replacement. It is not recommended for patients who simply refuse surgery on the basis of personal preference.

\section{Confirming the severity of aortic stenosis}

Currently, PAVI is only indicated in patients with calcified pure or predominant symptomatic aortic stenosis. The different imaging modalities can assist in the selection process by providing important information on the aortic valve, coronary arteries, and vascular structures. First, the severity of aortic stenosis should be assessed. Both transthoracic (TTE) and transoesophageal (TEE) Doppler echocardiography are the preferred tools to assess the severity of aortic stenosis. In addition, the exact anatomy of the aortic valve should be assessed. Echocardiography, multi-slice CT (MSCT) and magnetic resonance imaging (MRI) can all help to distinguish between a bicuspid and a tricuspid aortic valve. It is important to point out that implantation of available percutaneous prostheses is contraindicated in the case of a unicuspid or bicuspid aortic valve, because of the risk of incomplete deployment, significant paravalvular regurgitation, and displacement of the prosthesis $[5,6]$. A severely calcified aortic valve may result in the inability to cross the native valve with the catheter. Bulky leaflets and calcifications on the free edge of the leaflets may increase the risk of occlusion of the coronary ostia during aortic valve implantation. Therefore, the extent and exact location of calcifications should be carefully assessed before the implantation procedure. The assessment of coronary anatomy is also important in the selection process. Conventional coronary angiography should be performed to exclude the presence of significant coronary artery disease. Conventional angiography remains the "gold standard".

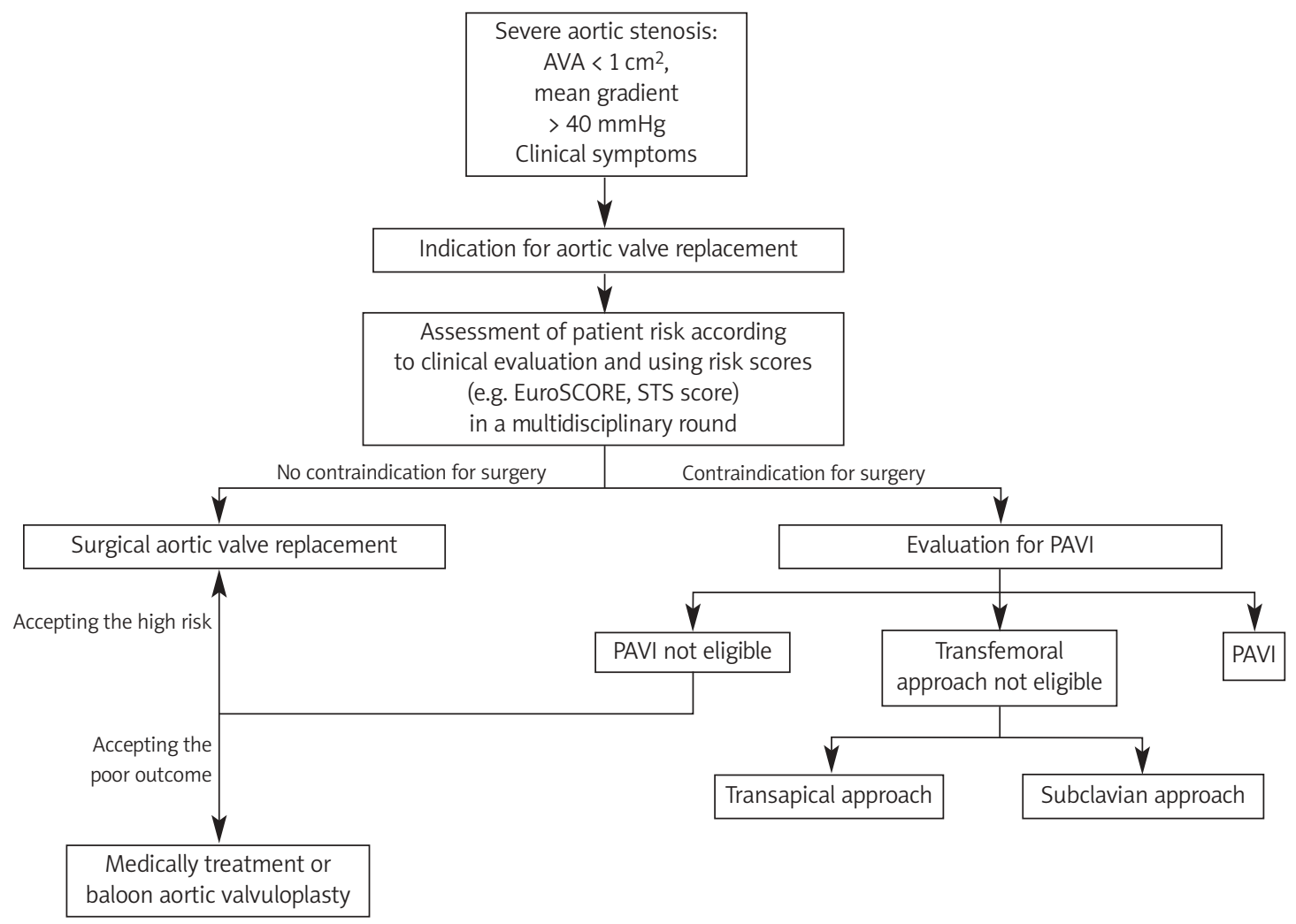

Figure 1. Algorithm to determine the treatment options of patients with severe aortic valve stenosis AVA - aortic valve area, $P A V I$ - percutaneous aortic valve implantation 


\section{Analysis of surgery risk and evaluation of life expectancy and quality of life}

The precise evaluation of surgical risk in a specific patient is not easy and involves an attempt at individualisation based on statistical data from databases containing a large number of procedures. The most accepted and validated algorithms that are widely available today are the EuroSCORE, the STS (Society of Thoracic Score) and the Parsonnet score. These algorithms predict the surgical risk by assigning weight to various factors that affect the clinical result, but it is clear that they can underestimate or overestimate it in certain groups of patients who are not represented satisfactorily in the population used to generate the algorithm [7]. There is some evidence in the literature of incorrect prediction of aortic valve replacement outcome using the EuroSCORE model [8]. Osswald et al. report on the real risk of overestimation of death by EuroSCORE for patients undergoing isolated aortic valve replacement, highlighting a possible incorrect assignment of high-risk patients to the PAVI procedure [9]. The key element to establish whether patients are at high risk for surgery is multidisciplinary clinical judgement, which should be used in association with a more quantitative assessment, based on the combination of several scores (for example expected mortality $>20 \%$ with the EuroSCORE and >10\% with STS score). This approach allows the team to take into account risk factors that are not covered in scores but often seen in practice such as chest radiation, previous aortocoronary bypass with patent grafts, porcelain aorta, liver cirrhosis, etc.

\section{Assessment of feasibility and exclusion of contraindications for percutaneous aortic valve implantation}

After criteria of severe symptomatic aortic valve stenosis and high surgical risk are evaluated, the technical evaluation of the patient's suitability for the percutaneous implantation technique begins (Table I). The two most basic parameters are the suitability of the peripheral arteries and the size of the aortic valve annulus. Contrast angiography is needed to assess the former, while the latter requires an initial assessment of the diameter of the aortic annulus on a TTE. In general terms, a large artery with dominant elastic elements should have a diameter up to $1 \mathrm{~mm}$ smaller than the external diameter of the sheath that has to be introduced for the valve implantation. Thus, current systems with an external sheath diameter of $28 \mathrm{~F}$ (SAPIEN 26 mm, Edwards), 25 F (SAPIEN 23 mm, Edwards) and $22 \mathrm{~F}$ (CoreValve, Medtronic) require minimum diameters in the order of $8 \mathrm{~mm}, 7 \mathrm{~mm}$ and $6 \mathrm{~mm}$, respectively. Apart from the minimum diameter, the existence of significant vessel

Table I. Currently proposed indications and contraindications for percutaneous aortic valve implantation

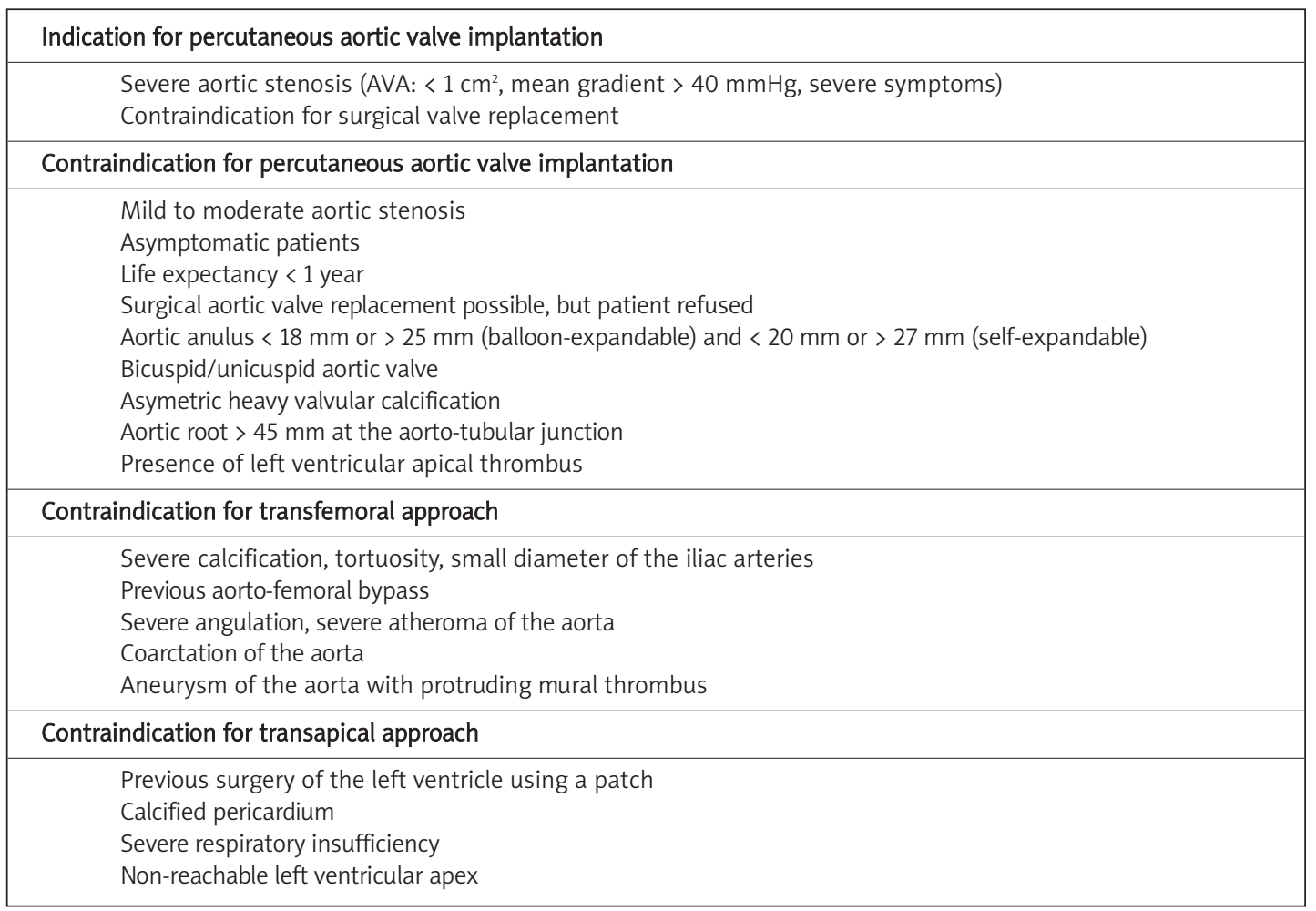


tortuosity $\left(>90^{\circ}\right)$, especially when combined with wall calcifications, makes advancing the large sheath problematic, with a high risk of vascular complications that could potentially affect the final outcome. In addition, the existence of extensive circumferential calcifications limits the elastic dilation of the artery; thus, the minimum diameters referred to above are underestimated. Patients who do not meet the criteria of suitable peripheral arterial access may still be candidates for transapical implantation. For the assessment of aortic annulus diameter we should keep in mind that TTE underestimates its size by a mean of $1.4 \mathrm{~mm}$ compared to TEE $[5,10]$, while the latter method also underestimates the size by $1.2 \mathrm{~mm}$ compared with intraoperative measurement [10]. Therefore, in order to avoid undesirable and often catastrophic displacement of the prosthesis, there should be a margin of at least 1-2 $\mathrm{mm}$ between the diameter of the valve and the size of the aortic annulus estimated using TEE, so that the former may be successfully and safely anchored within the latter. Computed tomography scan aortography and angiography of the ascending aorta are the most appropriate examinations for investigating these aspects. Those examinations will also be used for the measurement of the dimensions of the ascending aorta and the aortic arch, which are essential for checking eligibility for the CoreValve (the most important being the diameter of the ascending aorta, which should be $<4.3 \mathrm{~cm}$ ). The anatomy of the thoracic aorta (any chance of porcelain aorta) and the abdominal aorta should be studied by some imaging method for the existence of extensive atheromatosis, mural thrombi and aneurysm.

\section{Different transcatheter aortic valves}

On the basis of the first results from clinical trials, the CoreValve ReValving System and Edwards
Lifesciences System obtained the CE mark of approval in 2007 with the specification that these valves are intended for patients with a high or prohibitive risk for surgical valve replacement or who cannot undergo AVR. The first generation balloon expandable valve was called the CribierEdwards valve (Edwards Lifesciences Inc), whereas at present the Edwards SAPIEN valve (Edwards Lifesciences Inc) is commercially available (Figure 2). The Edwards Lifesciences SAPIEN THV device is a balloon-expandable valve. It consists of bovine pericardium that is firmly mounted within a tubular, slotted, stainless steel balloon-expandable stent. Two valve sizes have been developed $(23 \mathrm{~mm}$ and $26 \mathrm{~mm}$ ). At present, available prosthesis sizes are $23 \mathrm{~mm}$ and $26 \mathrm{~mm}$ for aortic annulus diameters in the range $18-22 \mathrm{~mm}$ and $21-25 \mathrm{~mm}$, respectively. The CoreValve ReValving device is a self-expanding frame-valve prosthesis (Figure 2). It consists of a porcine pericardial tissue valve that is mounted and sutured in a multilevel self-expanding nitinol frame. It is available in $26 \mathrm{~mm}$ and $29 \mathrm{~mm}$ sizes. The device has a broader upper segment (outflow aspect), which yields proper orientation to the blood flow. The first generation valve used bovine pericardial tissue and was constrained within a 25 French (F) delivery catheter. The second generation valve was built with porcine pericardial tissue within a $21 \mathrm{~F}$ catheter to allow access through smaller diameter vascular beds. The third generation of the device features a catheter with a valve delivery sheath size of $18 \mathrm{~F}$ and a follow-on shaft of 12 F. Newer devices that have first-in-man application include Paniagua (Endoluminal Technology Research, Miami, FL), Enable (ATS, Minneapolis, MN), AoTx (Hansen Medical, Mountain View, CA), Perceval (Sorin Group, Arvada, CO) Jena (JenaValve Technology, Wilmington, DE), Lotus Valve (Sadra Medical, Campbell, CA), and Direct Flow percutaneous aortic valve (Direct Flow Medical, Inc.,

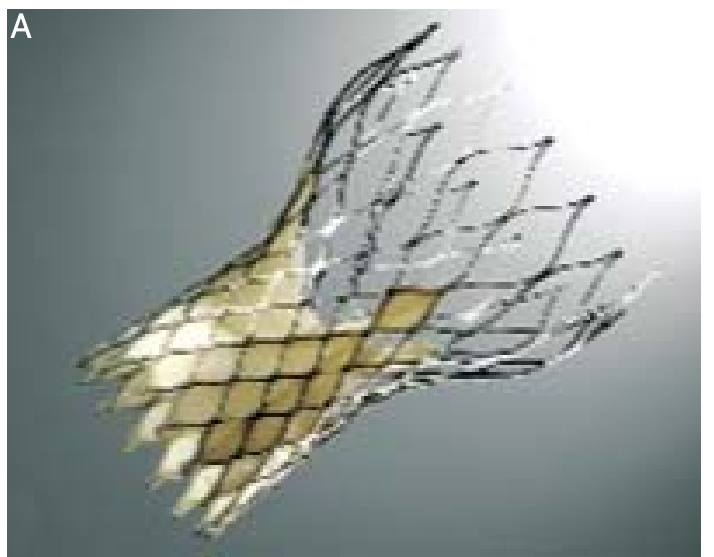

B

Figure 2. Profile of the Edwards SAPIEN Transcatheter Heart Valve (A) and CoreValve Revalving System (B) 
Santa Rosa, CA). Percutaneous aortic valve implantation represents a unique challenge for anaesthesiologists. As with other invasive procedures, a careful preoperative assessment, appropriate intraoperative monitoring and imaging, meticulous management of haemodynamics, and early treatment of expected side effects and complications are of utmost importance. An unexpected decrease or increase in systemic vascular resistance resulting in decreased coronary perfusion pressure or acute heart failure by elevated left ventricular end-diastolic pressure should be avoided by maintaining a normotensive blood pressure and heart rate between $60 \mathrm{bpm}$ and 100 bpm. The choice of anaesthetic technique, either local anaesthesia with mild sedation promoting spontaneous respiration, deep IV sedation with insertion of a laryngeal mask or general anaesthesia, varies among centres and is probably not associated with a significant difference in outcome. Post valvuloplasty and implantation, done under rapid right ventricular pacing to reduce left ventricular ejection and cardiac motion, may require some additional inotropic support. Tracheal extubation can usually be performed at the end of the procedure. Close postoperative monitoring is necessary, and admission to an intensive care unit is required. However, at present a retrograde approach through the femoral artery is used. During the procedure, balloon valvuloplasty is first performed to facilitate passage of the native aortic valve. During rapid right ventricular pacing, the prosthesis is positioned and deployed under fluoroscopy and echocardiographic guidance. Alternatively, in patients with difficult vascular access because of extensive calcifications or tortuosity of the femoral artery or aorta, a transapical approach can be used. After a partial thoracotomy, direct puncture of the apical portion of the left ventricular free wall is performed to gain catheter access to the left ventricle and aortic valve. The prosthesis is subsequently positioned and deployed, similar to the antegrade approach.

\section{Results from the literature}

\section{Cribier-Edwards valve}

Cribier et al. performed the first human implantation in 2002 [11]. In the Initial Registry of EndoVascular Implantation of Valves in Europe (I-REVIVE) trial, followed by the Registry of Endovascular Critical Aortic Stenosis Treatment (RECAST) trial, a total of 36 patients (mean [SD] EuroSCORE 12 [2]) were included [12] Twenty-seven patients underwent successful percutaneous aortic valve implantation (23 antegrade, 4 retrograde). The 30 -day mortality was $22 \%$ (6 of 27 patients), and the mean aortic valve area increased from 0.60 $\pm 0.11 \mathrm{~cm}^{2}$ to $1.70 \pm 0.10 \mathrm{~cm}^{2}$ ( $p<0.001$ ). Importantly, this improvement in aortic valve area was maintained up to 24 months follow-up [13]. After these first trials, the Cribier-Edwards prosthesis and the Edwards SAPIEN prosthesis have been used in numerous studies. Overall, acute procedural success is achieved in $75-100 \%$ of the procedures, and 30 -day mortality ranges between $8 \%$ and $50 \%$ in the published studies. Using the transapical technique and the SAPIEN valve, Walther has reported the initial multicentre results of 59 consecutive patients, which is the largest feasibility study published thus far. Procedural success using the transapical technique was achieved in 53 patients. Thirty-day mortality was $13.6 \%$ and none of these were thought to be valve related as there was good valve function at autopsy [14].

\section{CoreValve ReValving}

Since the first implantation of the CoreValve prosthesis in a patient in 2005 [15], a large number of patients have been treated with this device to date. The feasibility and safety of this valve was studied in a prospective, multicentre trial. A total of 25 symptomatic patients with an aortic valve area $<1 \mathrm{~cm}^{2}$ were enrolled in the study. The device was successfully implanted using the retrograde technique in 22 of 25 patients. Procedural success and aortic mean pressure gradients were markedly improved immediately following implantations, with pre-procedure gradients of $44.24 \pm 10.79 \mathrm{mmHg}$ to $12.38 \pm 3.03 \mathrm{mmHg}$ post-procedure, and were about the same at 30-day follow-up (11.82 $\pm 3.42 \mathrm{mmHg})$. NYHA class improved by 1 to 2 grades in all patients. Major adverse cardiac events (MACE; defined as death from any cause, major arrhythmia, myocardial infarction, cardiac tamponade, stroke, urgent or emergent conversion to surgery or balloon valvuloplasty, emergent percutaneous coronary intervention, cardiogenic shock, endocarditis, or aortic dissection) occurred in 8 of the 25 patients while in the hospital [16]. Recently, Grube et al. reported the results with the three different generations of the CoreValve ReValving system. In this non-randomised, prospective study, a total of 136 patients were included [17]. Ten patients were treated with first generation devices, 24 patients with second generation, and 102 patients with third generation devices. At baseline, mean aortic valve area was $0.67 \mathrm{~cm}^{2}$ and mean logistic EuroSCORE was $23.1 \%$ in the overall study population. Overall procedural success rate increased significantly with the new generation devices from $70.0 \%$ and $70.8 \%$ to $91.2 \%$ for the first, second, and third generation prostheses, respectively ( $p=0.003$ ). Interestingly, periprocedural mortality decreased using newer 
devices from $10 \%$ (first generation) to $8.3 \%$ (second generation) to $0 \%$ (third generation). Overall 30-day mortality for the three generations was 40\%, $8.3 \%$ and $10.8 \%$, respectively. Pooled data demonstrated a significant improvement in mean NYHA functional class (from 3.3 to 1.7, $p<0.001$ ), without a difference between the three generations. Importantly, NYHA functional class and mean pressure gradient remained stable up to 12 months follow-up in all three generations. In addition, the results of a multicentre registry with the third generation CoreValve ReValving system have recently been reported. A total of 646 patients from 51 centres were included in the registry. It was a high-risk elderly population (mean age 81 years) with a poor functional class ( $85 \%$ of the patients in NYHA class III or IV), and a high logistic EuroSCORE (mean $23.1 \%$ ). Procedural success was achieved in 628 of the 646 patients (97.2\%). All-cause 30-day mortality was $8 \%$, and the combined end point of procedural related death, stroke or myocardial infarction was reached in 60 patients (9.3\%). After successful implantation, mean pressure gradient decreased from $49 \mathrm{mmHg}$ to $3 \mathrm{mmHg}$ [18].

\section{Conclusions}

Percutaneous aortic valve implantation was developed in order to provide an alternative and less invasive method of treating aortic valve stenosis. It has now been proven that the method is feasible, with results that have been reproduced by many physicians in many centres (approximately 8000 implantations to date). Today there are at least 10 new percutaneous aortic valves that have had their first implantation in humans, many more that have reached the level of animal experiments, and even more that are still in the initial design stage. As a new treatment tool it has to be evaluated in randomised controlled trials with longterm follow-up in order to assess the safety and efficacy. Therefore, the performance of PAVI should be restricted to a limited number of high-volume centres, which have both cardiology and cardiac surgery departments, with expertise in structural heart disease intervention and high-risk valvular surgery. Due to the excellent results with surgical valve replacement, patient selection, which should be performed in multidisciplinary conferences, is of utmost importance. Like other interventional procedures, there is a learning curve with significant improvements in the success rate and the clinical results after the first 25 procedures, which implies that the PAVI procedure should be initially performed and thereafter supervised by a special team $[19,20]$. Beside the selection and intervention of PAVI, close follow-up with assessment of clinical and objective parameters is mandatory for defining the indications of this technique.

\section{References}

1. Nkomo VT, Gardin JM, Skelton TN, et al. Burden of valvular heart disease: a population-based study. Lancet 2006; 368: 1005-11.

2. Kvidal P, Bergstrom R, Horte LG, Stänk E. Observed and relative survival after aortic valve replacement. J Am Coll Cardiol 2000; 35: 747-56.

3. Iung B, Cachier A, Baron G, et al. Decision-making in elderly patients with severe aortic stenosis: why are so many denied surgery? Eur Heart J 2005; 26: 2714-20.

4. Varadarajan P, Kapoor N, Bansal RC, Pai RG. Clinical profile and natural history of 453 nonsurgically managed patients with severe aortic stenosis. Ann Thorac Surg 2006; 82: 2111-5.

5. Vahanian A, Alfieri O, Al-Attar N, et al. Transcatheter valve implantation for paients with aortic stenosis: a position statement from the European Association of CardioThoracic Surgery (EACTS) and the European Society of Cardiology (ESC), in collaboration with the European Association of Percutaneous Cardiovascular Interventions (EAPCI). Eur Heart J 2008; 29: 1463-70.

6. Zegdi R, Ciobotaru V, Noghin M, et al. Is it reasonable to treat all calcified stenotic aortic valves with a valved stent? Results from a human anatomic study in adults. J Am Coll Cardiol 2008; 51: 579-84.

7. Roques F, Nashef SA, Michel P; EuroSCORE Study Group. Risk factors for early mortality after valve surgery in Euro in the 1990 's: lesson from the EuroSCORE pilot program. J Heart Valve Dis 2001; 10: 572-8.

8. Grossi EA, Schwartz CF, Yu PJ, Jorde UP, Crooke GA, Grang B. High-risk aortic valve replacement: are the outcomes as bad as predicted? Ann Thorac Surg 2008; 85: 102-6.

9. Moss RR, Ivens E, Pasupati S, et al. Role of echocardiography in percutaneous aortic valve replacement. JACC Cardiovasc Imaging 2008; 1: 15-24.

10. Babaliaros VC, Liff D, Chen EP, et al. Can balloon aortic valvuloplasty help determine appropriate transcatheter aortic valve size? JACC Intv 2008; 1: 580-6.

11. Cribier A, Eltchaninoff $\mathrm{H}$, Bash A, et al. Percutaneous transcatheter implantation of an aortic valve prosthesis for calcific aortic stenosis: first human case description. Circulation 2002; 106: 3006-8.

12. Cribrier A, Eltchaninoff H, Tron C, et al. Early experience with percutaneous transcatheter implantation of heart valve prosthesis for the treatment of end-stage inoperable patients with calcific aortic stenosis. J Am Coll Cardiol 2004; 43: 698-703.

13. Cribrier A, Eltchaninoff H, Tron C, et al. Treatment of calcific aortic stenosis with the percutaneous heart valve: midterm follow-up from the initial feasibility studies: the French experience. J Am Coll Cardiol 2006; 47: 1214-23.

14. Walther T, Simon P, Dewey T, et al. Transapical minimally invasive aortic valve implantation: multicenter experience. Circulation 2007; 116 (Suppl 11): I240-5.

15. Grube E, Laborde JC, Zickmann B, et al. First report on a human percutaneous transluminal implantation of a self-expanding valve prosthesis for interventional treatment of aortic valve stenosis. Catheter Cardiovasc Interv 2005; 66: 465-9.

16. Grube E, Laborde JC, Gerckens U, et al. Percutaneous implantation of the CoreValve self-expanding valve prosthesis in high-risk patients with aortic valve disease: Siegburg first-in-man study. Circulation 2006; 114: 1616-24. 
17. Grube E, Buellesfeld L, Mueller R. Progress and current status of percutaneous aortic valve replacement: results of three device generations of the CoreValve Revalving system. Circ Cardiovasc Intervent 2008; 1: 167-75.

18. Piazza N, Grube E, Gerckens U, den Hejers P, Linke A. Procedural and 30-day outcomes following transcatheter aortic valve implantation using the third generation $(18 \mathrm{Fr})$ corevalve revalving system: results from the multicentre, expanded evaluation registry 1-year following CE mark approval. Eurolntv 2008; 4: 242-9.

19. Webb JG, Pasupati S, Humphries K, et al. Percutaneous transarterial aortic valve replacement in selected high-risk patients with aortic stenosis. Circulation 2007; 116: 755-63.

20. Walther T, Dewey T, Borger MA, et al. Transapical aortic valve implantation: stepp by stepp. Ann Thorac Surg 2009; 87: 276-83. 